\title{
Das Gedicht De Solis ac Lunae defectibus des Roger Boscovich (1711-1787): wissensvermittelnde Poesie in antiker und nachantiker Tradition
}

\section{Einleitung}

Als der in Ragusa geborene Universalgelehrte Roger Boscovich (1711-1787) ${ }^{1}$ im Jahre 1760 sein Lehrgedicht De Solis ac Lunae defectibus in London drucken lässt, ${ }^{2}$ kann der Text bereits auf eine 25 -jährige Produktions- und Rezeptionsgeschichte zurückblicken. ${ }^{3}$ Wie der damalige Leser im Vorwort erfährt, geht das monumentale Werk auf einen aus 300 Versen bestehenden Nucleus zurück, den der Autor zu Beginn des akademischen Jahres 1735 im Collegio Romano rezitiert hat. In den folgenden Jahrzehnten erfuhr der Text eine kontinuierliche Erweiterung, so dass verschiedene Fassungen im akademischen Milieu zirkulierten. Auch mit der 1760 erfolgten typographischen Publikation ist die durch mouvance geprägte Geschichte des Gedichts keineswegs abgeschlossen. Im Jahr 1761 wird der Text in Venedig gedruckt, 1765 in Graz, zudem erscheint er 1779 in Paris zusammen mit einer französischen Übersetzung. Dass die erste typographische Fassung gerade 1760 und gerade in London erscheint, versehen mit einem Lob auf Isaac Newton, resultiert aus der damaligen Situation des Autors: Nicht zufällig widmet der zu dieser Zeit für sieben Monate (Mai bis Dezember 1760) als Diplomat in London lebende Boscovich seinen Text der Royal Society, in die er dann folgerichtig kurz darauf, im Januar 1761, aufgenommen wird.

Das in der Londoner Ausgabe insgesamt 5.508 Hexameter umfassende, mithin epische Ausmaße zeigende Lehrgedicht behandelt in fünf Büchern die

$1 \mathrm{Zu}$ seinem Leben vgl. einleitend Paolo Casini: Boscovich, Ruggero Giuseppe. In: Dizionario Biografico degli Italiani 13 (1971), S. 221-230; Piers Bursill-Hall (Hg.): R. J. Boscovich: Vita e attività scientifica. His Life and Scientific Work. Rom 1993, hierin insbes. Sante Graciotti: Le idee e l'arte del letterato Boscovich, S. 27-39.

2 Rogerius Iosephus Boscovich: De solis ac lunae defectibus libri V. London 1760 (nach dieser Ausgabe wird im Folgenden zitiert).

3 Zur Entstehungs- und Druckgeschichte vgl. Žarko Dadić (Hg.): Edizione Nazionale delle opere e della corrispondenza di Ruggiero Giuseppe Boscovich. De Solis ac Lunae defectibus. Rom 2012, S. 11-21 (Einleitung; die Edition selbst beinhaltet nur einen photomechanischen Nachdruck des frühneuzeitlichen Drucks). 
Sonnen- und Mondfinsternis sowie einige weitere Aspekte der Astronomie und Optik. ${ }^{4}$ Im ersten Buch führt Boscovich den Leser in die Astronomie ein und erklärt im Rahmen einer Synopse die Bewegung der Sterne (985 Verse sowie ein separat gezähltes Additamentum von 78 Versen). Das zweite Buch (1.426 Verse) beschäftigt sich grundsätzlich mit dem Thema der Sonnen- und Mondfinsternis. Im dritten Buch (1.035 Verse) legt der Dichter einzelne Effekte und Phänomene dar, welche während einer Sonnen- oder Mondfinsternis auftreten. Das vierte Buch (960 Verse) knüpft hieran an und erläutert ausführlich den während der Mondfinsternis $\mathrm{zu}$ beobachtenden Erdschatten. Im fünften Buch (1.024 Verse) stellt Boscovich Isaac Newtons Erkenntnisse zur Gravitation und Optik vor.

Dass ein Mann wie Boscovich, der seinen Zeitgenossen als wissenschaftliches Wunderkind gegolten hat und schon mit 29 Jahren zum Professor für Mathematik berufen worden ist, bei der Behandlung aktueller astronomischer Fragen auch die poetische Form für ein in akademischen Kreisen legitimes Ausdrucksmittel hält, ist angesichts seines persönlichen und sozialen Umfeldes nicht überraschend. Wie Yasmin Haskell gezeigt hat, galt das Dichten in lateinischer Sprache in jesuitischen Milieus dieser Zeit und insbesondere in Rom, wo Boscovich lehrte, als eine natürliche Ausdrucksform, die so selbstverständlich war wie das Atmen. ${ }^{5}$ In Kombination mit dem jesuitischen Bildungsauftrag musste dieser Umstand dazu führen, dass insbesondere die Gattung des Lehrgedichts intensiv gepflegt wurde. ${ }^{6}$ Und schließlich kam hinzu, dass die Astronomie (inklusive der Astrologie) so fest wie keine andere Disziplin seit der

4 Zusammenfassung bei Dadić (Anm. 3), S. 12-20.

5 Yasmin Annabel Haskell: Loyola's Bees: Ideology and Industry in Jesuit Latin Didactic Poetry. Oxford 2003, hier insbes. S. 192. Vgl. ferner Diane Bitzel: Bernardo Zamagna, Navis Aëria. Eine Metamorphose des Lehrgedichts im Zeichen des technischen Fortschritts. Frankfurt a. M. u. a. 1997 (Studien zur klassischen Philologie 109), S. 12-14. Zum jesuitischen Hintergrund vgl. auch die klugen Überlegungen bei Luca Guzzardi (Hg): Edizione nazionale delle opere e della corrispondenza di Ruggiero Giuseppe Boscovich. Vol. XIII/2: Les Eclipses. Poëme en six chants. Rom 2012, S. 11-16. Zu Boscovichs Gelegenheitsgedichten vgl. zuletzt Nada Savkovic: Occasional Poetry by Roger Boscovich. In: Almagest. International Journal for the History of Science 6,1 (2015), S. 32-47.

6 Zur neulateinischen Lehrdichtung vgl. Yasmin Haskell: The Classification of Neo-Latin Didactic Poetry from the Fifteenth to the Nineteenth Centuries. In: Brills' Encyclopaedia of the Neo-Latin World. Hg. von Philip Ford, Jan Bloemendal, Charles Fantazzi. Bd. 1-2. Leiden, Boston 2014, S. 437-448; Heinz Hofmann: Aristaeus und seine Nachfolger: Bemerkungen zur Rezeption des Aristaeus-Epyllions in der neulateinischen Lehrdichtung. In: Humanistica Lovaniensia 52 (2003), S. 343-398; Yasmin Haskell, Philip Hardie (Hg.): Poets and Teachers: Latin Didactic Poetry and the Didactic Authority of the Latin Poet from the Renaissance to the Present. Bari 1999 (mit Bibliographie S. 15-19). Zu französischen Dichtungen vgl. Isabelle Pantin: La poésie du ciel en France dans la seconde moitié du seizième siècle. Genf 1995. 
Antike (durch Hesiod, Arat, Lukrez, Vergil, Manilius u. a.) in der Lehrgedichtstradition etabliert war. Das gesamte Mittelalter und auch die italienische Renaissance (Giovanni Pontano, Basinio da Parma, Lorenzo Bonincontri u. a.) haben diese Tradition weitergepflegt. Ein literarisch ambitionierter Autor der Frühen Neuzeit konnte sich der machtvollen und herausfordernden poetischen Konvention somit kaum verweigern. ${ }^{7}$ Im Gegenteil: Der Wunsch, sich mit den antiken und den zeitgenössischen Gattungsvertretern (Carlo Noceti, Orazio Borgondio, Benedetto Stay) nicht nur wissenschaftlich, sondern auch literarisch zu messen, dürfte ein wichtiger Ansporn gewesen sein.

\section{Die Vorrede des Werkes}

Obwohl die moderne Forschung Boscovich nicht nur als bedeutenden Wissenschaftler feiert, sondern auch seine poetischen Fähigkeiten anerkennt, ist das Gedicht De Solis ac Lunae defectibus unter literarischer und texttypologischer Fragestellung bislang nicht näher untersucht worden. Dabei ist die Quellenlage zur Analyse seines poetischen Konzeptes recht günstig, da der Autor dem Lehrgedicht eine umfangreiche Prosa-Praefatio voranstellt. Hier heißt es einleitend (Ad Lectorem, S. i):

Opus inscribitur De Solis, ac Lunae defectibus, quod quidem argumentum videtur prima fronte maximè aridum, et jejunum; et quidem fuisset ejusmodi, nisi, occasione arrepta, plurima, quae aliquem cum eo nexum habent, pertractanda suscepissem.

Der Autor konzediert hier in bester Lehrgedichtstradition, dass der behandelte Stoff auf den ersten Blick trocken und fade erscheinen könne. Er dementiert dieses grundsätzliche Faktum nicht, verweist aber darauf, dass es durch literarische Gegenmaßnahmen abgefedert sei: Um die sachliche Darstellung aufzulockern, habe er, so Boscovich, an geeigneten Stellen zahlreiche Abschnitte eingefügt, die zwar eine Beziehung zum Hauptthema aufwiesen, jedoch den

7 Zur astronomischen Lehrdichtung in der Frühen Neuzeit vgl. Yasmin Haskell: Renaissance Latin didactic poetry on the stars: wonder, myth, and science. In: Renaissance Studies 12 (1998), S. 495-522; Wolfgang Hübner: Die Rezeption des astrologischen Lehrgedichts des Manilius in der italienischen Renaissance. In: Humanismus und Naturwissenschaften. Hg. von Rudolf Schmitz, Fritz Krafft. Boppard 1980 (Beiträge zur Humanismusforschung 6), S. 39-67; Walther Ludwig: Neulateinische Lehrgedichte und Vergils Georgica. In: From Wolfram and Petrarch to Goethe and Grass: Studies in Literature in Honour to Leonard Forster. Hg. von D. H. Green u. a. Baden-Baden 1982 (Saecula Spiritalia 5), S. 151-180, hier S. 151 f. u. 155. 
auf Dauer ermüdenden Erklärungsmodus unterbrächen. Was hiermit gemeint ist, erklärt der Autor im weiteren Verlauf der Vorrede (Ad Lectorem, S. ii):

Haec ad argumentum pertinent, quibus episodia immiscui sane multa, ad demulcendum animum severiorum contemplatione defatigatum, quorum episodiorum praecipua in omnium librorum fine invenies.

Vor allem am Ende der einzelnen Bücher hat der Dichter somit längere Digressionen eingefügt, die bei der Lektüre eine geringere Konzentration erfordern. Bei diesen episodia handelt es sich um weitere astronomische und optische Darlegungen, ferner inseriert Boscovich rhetorische Apostrophen (an Bradley und Newton) und mythologische Elemente. Der Einsatz dieses - immerhin paganen - Mythos wird von dem jesuitischen Autor ausdrücklich gerechtfertigt (Ad Lectorem, S. ii):

Veterum itidem mythologia fere ubique sum usus, cum de astris agerem, quibus et vitam, et vero etiam Divinitatem ii dederant, quod ad amoenitatem carminis opportunum fore censui, nec vero ineptum ad profundiora etiam tanquam mysteria facilius ingerenda, et explicanda.

Boscovich verweist hier erstens auf die antik-pagane Tradition, welche den Planeten eine anthropomorphe Göttlichkeit zugeordnet hat. Zweitens dient ihm der Mythos als poetisches Ornament. - Insbesondere durch das rhetorische Mittel der Personifikation kann die sachliche Darstellung lebendiger und interessanter gestaltet werden. Drittens erscheint die mythologische Überformung auch deshalb gerechtfertigt, weil hier (in der Astronomie) gleichsam göttliche Geheimnisse offenbart und erklärt werden.

Selbstverständlich erläutert der Dichter in der Vorrede auch den Aufbau und Inhalt seines Werkes. So schreibt er bezüglich einer Refraktionstheorie, die er im vierten Buch darlegt (Ad Lectorem, S. ii):

[...] theoriam autem proposui distributionis hujusce refracti luminis per umbram Terrae, quam ad eclipseos Lunaris cognitionem maxime pertinere arbitror, quam quidem alibi nequaquam inveni.

Boscovich weist den Leser somit ausdrücklich darauf hin, dass man die Präsentation bzw. Erläuterung dieser Theorie in keinem anderen Werk, d. h. weder in einem Lehrgedicht noch in einem Prosa-Traktat, finde. Ähnlich heißt es zu einem Thema des fünften Buches (Ad Lectorem, S. ii):

Quinto demum libro illud exposui, cur Luna in totalibus eclipsibus saepe admodum appareat rubro colore affecta, cujus itidem phaenomeni satis uberem explicationem alibi non reperi [...].

Der Autor arbeitet hier keineswegs mit dem klassischen und für das Genre üblichen primus-inventor-Motiv: Er ist stolz darauf, dieses Thema nicht etwa als 
Erster im Rahmen der Lehrdichtung, sondern als Erster überhaupt behandelt zu haben. Seine Leistung ist daher eine primär wissenschaftliche, und nur zusätzlich auch eine poetische!

Im weiteren Verlauf erzählt Boscovich die lange Geschichte der Genese und Entwicklung seines vielschichtigen Textes. Den Nucleus bildete eine dreihundert Verse umfassende Partie, die er bereits 1735 im Collegium Romanum mündlich vortrug. ${ }^{8}$ Doch erst als er sich zwölf Jahre später mit der Lehrdichtung des Carlo Noceti befasst habe, sei die eigene Poesie wieder in den Fokus gerückt. ${ }^{9}$ Danach habe er, so der Autor, zwischen 1747 und 1760 an verschiedenen Stellen viele weitere Partien hinzugefügt, ${ }^{10}$ eine Drucklegung des gesamten Werkes jedoch immer wieder verschoben. ${ }^{11}$ Erst jetzt veröffentliche er das Gedicht zu Ehren der Royal Society (welche als Widmungsnehmer auftritt). ${ }^{12}$ Allerdings würden bereits unter den Freunden in Italien, Deutschland und Frankreich handschriftliche Fassungen kursieren, die den Text vollständig oder in Auszügen überlieferten und in Details von der jetzigen Druckversion abwichen. ${ }^{13}$ Und wie Boscovich andeutet, ist die Geschichte des Textes auch jetzt

8 Vorrede (Ad Lectorem), S. iii: „Plures occurrunt in ipso poëmate epochae pertinentes ad ea tempora, quibus certae ejus partes conscriptae sunt, quae non penitus inter se consentiunt. Et quidem fere casu quodam id in opus ordinatum coaluit. Conscripseram, et in solemni studiorum instauratione in Collegio Romano recitaveram jam ab anno 1735 poëmation de Solis, ac Lunae defectibus, quod tunc quidem trecentis circiter versibus continebatur totum: ii fere omnes nunc etiam remanent hac, illac dispersi primo, et secundo libro, et ipsum exordium cum prioribus binis invocationibus, et bina episodia, quae nunc habentur in eorum librorum fine, conservavi integra, paucis adjectis. Diu autem deinde jacuit, cum Theologicis primum, tum Mathematicis distractus studiis poësim fere penitus seposuissem.“

9 Vorrede (Ad Lectorem), S. iii: „Verum post duodecim circiter annos ad Musarum amorem sum revocatus exemplo hinc P. Caroli Noceti, inde Benedicti Stay, quorum mentionem hìc facio in annotationibus, qui philosophica argumenta versibus latinis elegantissimis persequebantur, quibus ego adnotationes et dissertationes adjeci plures, cum ederentur. Hinc ad ipsum, quod olim pertractaveram, argumentum revocavi animum [...].“

10 Vorrede (Ad Lectorem), S. iv: „Sunt autem nonnulla etiam multo posterius adjecta, ut ea, quae post finem libri primi occurrunt in laudem binorum Cardinalium, qui ad Arcadum Romanorum coetum, cum ejus libri initium ibi recitarem, advenerant, et initio ejusdem libri invocatio ad Regiam Societatem Londinensem, quam hìc in Anglia hoc anno elucubravi.“

11 Vorrede (Ad Lectorem), S. iv: „Editionem, quam plures amici, et Typographi postularent, tamdiu distuli [...].“

12 Vorrede (Ad Lectorem), S. v: „[...] et Regiae Societatis nomine insignitum opus emitto: nullam vero nuncupatoriam epistolam addo, cum ipsa invocatio, quae occurrit lib. I. ver. 19, et ejus adnotatio vices ipsius gerant.“

13 Vorrede (Ad Lectorem), S. v: „Extant apud amicos descripta exempla in Italia, Germania, Gallia vel totius operis, vel libri cujuspiam, a quibus nonnulla hìc identidem occurrent immutata, non quidem fusiora loca, sed plerumque voces, et hemistichia [...].“ 
keineswegs abgeschlossen: Aus Zeitmangel habe er bei der Vorbereitung der typographischen Ausgabe einiges ausgelassen, was er vielleicht später ergänzen werde. ${ }^{14}$ - Aus philologischer Sicht handelt es sich bei diesem Lehrgedicht somit um ein wunderbares Beispiel für die Möglichkeiten medialer, performativer, konzeptioneller, technischer und textueller mouvance: Das proteische Gedicht changiert zwischen Mündlichkeit und Schriftlichkeit, zwischen Vortrag und Fachbuch. Neue Textpartien werden hinzugefügt und alte umgestellt, einzelne Verse ändern ihre Gestalt. Es existieren verschiedene Fassungen nebeneinander, und solange der Autor lebt, kann es keine finale Version geben.

Nach dem eigenem Verständnis des Verfassers entspricht die Komposition solcher Lehrgedichte nicht der Arbeitsplatzbeschreibung eines Mathematikprofessors (oder eines Diplomaten). Boscovich betont daher, dass er solche Verse nur in seiner Freizeit (ocium) ${ }^{15}$ und nur zum eigenen Vergnügen komponiere (Ad Lectorem, S. v): [...] poësim tamen identidem ob animi oblectamentum quoddam tantummodo excoluit, non ex officio exercuit. Ob man eine solche Bescheidenheitsgeste ernstnehmen oder in den Bereich unaufrichtiger Topik verweisen soll, bleibt dahingestellt. ${ }^{16}$

Eine weitere Information, die für die Lektüre des nachstehenden Gedichts von eminenter Bedeutung ist, erhält der Leser ebenfalls bereits in der Vorrede (Ad Lectorem, S. ii):

Adnotationes adjeci, in quibus soluta oratione exposui dilucidius aliquanto, quae versibus pertractantur, ut ab iis etiam, qui in Astronomia, et universa Mathesi sunt rudes, facilius intelligi possent ipsa carmina.

Der Verfasser selbst hat seinem Text somit zum besseren Verständnis einige Prosa-Anmerkungen beigegeben, in denen der Inhalt der Verse erläutert wird. Diese Annotationen richten sich angeblich an solche Leser, die nicht über hinreichende Kenntnisse der Mathematik und Astronomie verfügen. In der typographischen Ausgabe erscheinen die Anmerkungen als Fußnoten. Ihre Anzahl ist erheblich: Im ersten Buch findet man 54, im zweiten 89, im dritten und vierten jeweils 49 und im fünften 78. Sie sind häufig sehr umfangreich und ziehen

14 Vorrede (Ad Lectorem), S. ii-iii: „Cogitabam et supplementa quaedam adjicere, in quibus geometria, et calculo in subsidium vocatis, omnia fusius exponerentur, et accuratius definirentur; sed ob tantam argumenti, quod amplexus sum, amplitudinem, nimis in longum abibat res, nec mihi advenae, ac paucis mensibus in Anglia commorato, et plurimis, quae cognoscenda erant, distracto, satis ocii ad id fuit, quod tamen nec omnino necessarium arbitratus sum. Addam id fortasse aliquando, si vita supererit.“

15 Vorrede (Ad Lectorem), S. iii: „,...] satis ocii ad id fuit [...].“

16 Als Antwort auf die Frage nach der Motivation jesuitischer Lehrdichtung notiert Haskell (Anm. 5), S. 205: „because they could“. Zum Aspekt der Freizeitbeschäftigung vgl. ebd., S. 206. 
sich über mehrere Seiten hin. Betrachtet man nur die Zahl der Wörter, so machen die Fußnoten wohl knapp die Hälfte des aus Vers und Prosa bestehenden Gesamttextes aus. Über die Fußnotenzeichen sind die beiden Teile zudem fest miteinander verklammert. Vor diesem Hintergrund ist es also eigentlich unzutreffend, das 1760 im Druck erschienene Buch De Solis ac Lunae defectibus als Lehrgedicht zu bezeichnen. Es handelt sich vielmehr um einen typologischen Mischtext, welcher aus Sachgedicht und Fachprosa besteht. Boscovich löst die dem Genre inhärente Spannung zwischen res und carmen zumindest teilweise dadurch auf, dass er die poetische Sprache der Verse durch die Fachsprache der Prosa ergänzt und erläutert.

Als Dichter hegt er literarische Ambitionen: Er beschränkt sich nicht auf eine brutale Metrifizierung des Stoffes und der Fachtermini, sondern bemüht sich um eine echte Poetisierung. Da dieses Verfahren zu Auslassungen sowie inhaltlichen und terminologischen Unschärfen führen muss, bietet Boscovich seinen Lesern zur Kompensation die Fußnoten an. Tatsächlich enthalten seine Anmerkungen umfangreiche Erklärungen in Fachprosa sowie weitere Elemente, die sich einem Metrum verweigern (Listen, Zahlen, mathematische Formeln etc.), ferner einige Stellenbelege und Verweise auf andere Dichter. Der poetische Text wird somit von Prosa-Texten nicht nur flankiert, sondern geradezu eingehegt. ${ }^{17}$

Anders als Boscovich es uns glauben machen will, wenden sich die Fußnoten daher keineswegs nur an fachlich weniger gebildete Leser. Sie sind keineswegs nur ,subsidiäres Beiwerk', sondern halten auch für den hard-core-Mathematiker wichtige Informationen bereit. Die Behauptung dient also offenbar nur dazu, die partielle Kapitulation des Dichters vor einer mitunter unlösbaren Aufgabe zu bemänteln. Boscovich könnte die üblichen Ausreden und Eskapismen der Lehrdichter bemühen, doch ist er viel zu sehr Wissenschaftler, als dass er sich erlaubt hätte, die sachlichen Ungenauigkeiten seiner Verse unkommentiert zu lassen. Dass er die mitunter schiefe Poesie durch Prosa begradigt, hängt zweifellos auch mit seiner tatsächlichen Zielgruppe zusammen: Das Buch richtet sich vor allem an Wissenschaftler und wissenschaftlich vorgebildete Personen, nicht etwa an ,Laien“.

\section{Die poetische und didaktische Umsetzung}

Angesichts der Erläuterungen des Vorwortes ist es nicht überraschend, dass der Leser schon gleich am Anfang des Gedichts mit Prosa konfrontiert wird: Im ersten Vers des Textes (I 1: Cur quondam aetheras Titan sine nube per auras)

$17 \mathrm{Zu}$ den sog. Paratexten vgl. ebd., S. 203. 
setzt Boscovich bereits beim zweiten Wort (quondam) eine Fußnote, in der er die Makrostruktur des Textes erläutert. ${ }^{18}$ Eine solche Anmerkung nützt keineswegs nur dem mathematisch weniger gebildeten Rezipienten, wie der Autor im Vorwort behauptet hat, sondern allen Lesern. Im weiteren Verlauf des ersten Buches streift Boscovich das dritte Keplersche Gesetz (lex) und notiert hierzu (I 487-493):

\section{[...]}

Quam tamen ipse quidem culto depromere versu, Haud ausim, et Latiis sperem celebrare Camoenis.

Namque mihi haud animo id subeat, nimis impare nisu

Vatibus ut vetita, et Musis impervia quaeram

Ire loca, ad Pindum transcurrere: talia tentet

Communis patriae decus, atque aeterna Ragusae

Gloria Stayades [...].

Der Dichter bekundet hier seine Unfähigkeit, dieses und die beiden anderen komplizierten Keplerschen Gesetze in Versen darzustellen. Allenfalls sein berühmter Landsmann Benedetto Stay sei hierzu in der Lage. In einer dazu gehörenden Fußnote unterstreicht Boscovich seine eigene poetische Teilkapitulation (S. 21, Anm. 29): Hae leges [...] admodum difficulter possunt exprimi nitido Latino versu potissimum hoc stylo amoeniore aliquanto, ac minus rigido, quem adhibere curavi [...]. Der Gegenstand ließe sich möglicherweise durch eine brutale Hexametrisierung in Verse fassen, doch nicht auf dem hohen poetischen Niveau, um das sich Boscovich bemüht. Daher begnügt er sich hier mit einer Auslassung.

Im vierten Buch beklagt der Autor erneut die Grenzen des poetischen Mediums (IV 257-262):

Sed quoniam nodis, et dura compede vincti

Heu miseri vates adstringimur, atque notarum

Usus abest, numeros versu nec possumus omnes

Exprimere, et rerum mensuras dicere certas;

Pauca tibi expediam, quae multum oratus Apollo,

Parnassique sinent leges, et dura Camoena.

Als Dichter unterliegt Boscovich vielen formalen Zwängen (nodis und compede), zudem kennt die Poesie keine geometrischen Formeln und mathematischen Zeichen (notarum), auch lassen sich nicht alle Zahlen (numeros) und

18 S. 1, Anm. 1: „Innuuntur hìc praecipua capita pertractanda in toto opere: porro in eclipsi totali Solis solent et stellae apparere: Luna autem dum deficit, aliquando ita est nigra, ut etiam ex oculis penitus evanescat, aliquando est admodum rubra.“ 
Maße (mensuras) in Hexametern ausdrücken. Aus diesem Grunde kann der Autor das hier behandelte astronomische Phänomen nur unzureichend beschreiben. Entsprechend heißt es in einer dazu gehörenden Fußnote (S. 164, Anm. 13):

Iterum hìc jure queror de difficultate exprimendi versibus res geometricas, et calculos; conor tamen praestare, quantum per poësim licet, praecipua attingens capita [...].

Die Anmerkung offenbart eine poetische Teilkapitulation: In der mathematischen Spitzenforschung, zu deren Repräsentanten auch der Autor selbst zählt, stößt Dichtung an ihre Grenzen. ${ }^{19}$

Allerdings sind Boscovichs Fußnoten nicht immer wissenschaftlicher Natur. So erwähnt er im zweiten Buch, dass er, im 42. Lebensjahr stehend, darauf hoffe, eine Forschungsreise zu den Irokesen, d. h. nach Nordamerika, unternehmen zu können (II 276-281):

Fors et ego Irroquiûm sedes, et inhospita regna,

Si tantum fortuna sinet, si vita superstes,

(Namque quid hae vires, quid non haec spondeat aetas,

Dum bis vicenis alter vix additur annus?)

Transcurram procul, et doctis comes ibo catervis.

Spero equidem [...].

In einer Anmerkung notiert der Autor dazu (S. 59, Anm. 15):

Anno 1742 [wohl Druckfehler statt 1752 - Th. H.], quo haec scripsi Octobri mense, inchoaveram tantummodo annum 42, natus 18 Maji anni 1711. Est autem quadragesimus secundus annus is, qui additur alter, sive secundus, bis vicenis, sive 40 .

Er erläutert hier, dass diese Textpassage im Jahre 1742 (recte: 1752) entstanden sei. Geradezu umständlich erklärt er zudem, wie die lateinische Umschreibung des zweiundvierzigsten Lebensjahres zu verstehen sei. Ein solcher philologischer Hinweis gehört allerdings eher in den latinistischen Anfangsunterricht. Man sieht hier, dass die Endredaktion des im Jahre $1760 \mathrm{zu}$ druckenden Buches in großer Hast erfolgt ist.

In seinen Versen erweist sich Boscovich als ein eleganter Poet, der mit den Konventionen des Lehrgedichts bestens vertraut ist. Das erste Buch wird durch

19 Eine weitere Klage findet man in IV 571-578: „At qua se densam lux aëre flexa per umbram // Distribuat lege, et Phoeben perfundat opacem, // Proh quantus latiis labor est efferre Camoenis! // Cerata rursum tela, calamoque nigranti // Esset opus, mollive inscriptis littore sulcis. // Tentabo tamen, et nervos, plectrumque sonantem // Expediam, duro saltem concludere versu // Si multa, si forte sinat prece victus Apollo.“ 
einen Prolog (I 1-57) eröffnet, dessen einleitende Verse mehrere gattungstypische Elemente aufweisen (I 1-9):

Cur quondam aetheras Titan sine nube per auras

Dum micat, et puro respergit lumine terras,

Praetexat subita nitidos ferrugine vultus,

Impatiensque morae fuscis nox prodeat alis

Atra die medio, stellasque inducat Olympo;

Cur, sudo dum laeta nitet Latonia Coelo,

Nunc tenebris condat caput, et nunc sanguine multo

Inficiat frontem, Latiis memorare Camoenis

Aggrediar, penitusque imas educere causas.

Der Autor beschreibt hier das Thema seines Werkes mit dem etablierten Instrument der indirekten Frage (Cur - Cur), ${ }^{20}$ er benennt ferner das gewählte Ausdrucksmedium (Latiis [...] Camoenis, in Abgrenzung zur Prosa) und benutzt mit penitusque imas educere causas eine Formel, die direkt auf das Genre der (sc. naturwissenschaftlichen) Lehrdichtung verweist. Auch die sich unmittelbar anschließende Invokation folgt den texttypologischen Konventionen (I 10-14):

Tu mihi, qui Arcadico, aethereo qui summus Olympo,

Phoebe, nites, naturae aditus tu pande repostos,

Divinumque immitte jubar; tu suffice venam

Te dignam: tua res agitur, seu lumina terris

Ipse neges, solita seu fraudes luce sororem.

Wie in der astronomisch-astrologischen Lehrdichtung üblich, wird Phoebus hier in seiner Doppelfunktion als Musengott, dem Boscovich als Angehöriger der Accademia degli Arcadi verpflichtet ist, und als wichtigster Stern, der eines der beiden zentralen Themen des Gedichts bildet, angerufen. ${ }^{21}$ Eine zweite Invokation zielt auf Urania (I 15-18), eine dritte auf George Parker, den Earl of Macclesfield, welcher damals den Vorsitz der Royal Society innehatte. ${ }^{22}$ Der poetische Prolog endet mit einer topischen Bitte um wohlwollende Aufnahme des Werkes. ${ }^{23}$

20 Daneben finden sich im Text auch zahlreiche direkte Fragen, so z. B. I 788-791: „Unde tamen cursus dubii discrimina tanta, // Erroresque Deae, trepidusque per aethera motus? // Accipe, temporibus quae longum ignota vetustis // Dî dederunt tandem, et nostri labor extudit aevi.“

21 Vgl. auch II 1244-1246: „[...] // Accipe, quod facili praesentes numine Musae // Expediunt, Pindique pater de vertice Phoebus // Ipse suo pandit plectro, fidibusque canoris.“

22 I 19-21: „Ante tamen Pindi patrem, doctasque sorores // Te primum mea vota vocant, te in carmina poscunt, // Nobile Parkeridum germen [...].“

23 I 55-57: „Ergo opus excipite, atque aures adhibete canenti, // Tuque, et tota cohors, facilemque adjungite mentem, // Ac trepidum assensu vatem firmate secundo.“ 
Hierauf setzt der Hauptteil des Gedichts ein (Vers I 57 ist zur Markierung kursiv gedruckt). In ihm finden sich alle Merkmale, die für das Genre typisch sind. So wird beständig der (potentielle) Informationsbedarf des Publikums erwähnt, ${ }^{24}$ ferner durch die Programmwörter nosse und causae und latentes die Tradition naturwissenschaftlicher und naturphilosophischer Poesie evoziert. ${ }^{25}$ Boscovich wendet sich immer wieder direkt an den Leser und steuert dessen Konzentration durch Imperative, Adhortative und rhetorische Fragen. ${ }^{26}$ Unter den dabei verwendeten Verben ragen discere und docere deutlich heraus. ${ }^{27}$ Der unterweisende Gestus des Lehrdichters zeigt sich etwa in folgendem Beispiel (II 37-38 u. 41-42):

His animadversis, age jam, causasque latentes

Contemplare vigil mecum, resque ordine disce:

[...]

Accipe; nam coeptis praesens aspirat Apollo,

Et faciles ultro veniunt ad carmina Musae.

Innerhalb seiner Poesie nimmt Boscovich keine ausdrückliche Profilierung des angesprochenen Lesers vor. Es bleibt unklar, ob das primäre Zielpublikum aus mathematisch vorgebildeten Wissenschaftlern, aus dichtungsaffinen Intellektuellen oder, wie bei vielen anderen Lehrgedichten, aus jungen Schülern und Studenten bestehen soll. Nur in der an Isaac Newton gerichteten Apostrophe heißt es eindeutiger (V 39-41):

Omnibus haec ignota olim mortalibus, acri

Vidisti ingenio primus: nunc te duce molles

Jam pueri in ludis, primoque docentur ab aevo.

24 Als Vorbild vgl. z. B. Ovid, Ars I 1-2: „Si quis in hoc artem populo non novit amandi, // hoc legat [...].“

25 Vgl. z. B. I 58-61: „Defectus Solis varios, Lunaeque labores // Si penitus nosse, et caecas evolvere causas // Est animus; superas scrutari ante omnia sedes // Cura sit, et positus astrorum, et discere motus“; I 862-863: „Haec etiam noscenda prius, si nosse latentes // Est animus rerum causas, Lunaeque labores // [...]“; III 59-60: „Horum principia, et causas si nôsse latentes // Quasque cupis; primum expediam [...].“

26 Vgl. z. B. II 50-51: „Ac tibi ne dubiam teneat sententia mentem, // Perlege [...]“; II 676: „Quid dubitas? [...]“; III 225: „Nonne vides [...]? [...]“; III 317-318: „Quae si perpendas simul omnia, concidet error // Deprensus [...]“; IV 407 u. 409: „Ac tibi ne dubiam teneat sententia mentem, // [...] // Et radii perpende viam [...].“

27 Vgl. z. B. II 839-840: „[...] // Quod superest, tanti pars et quae maxima Divae // Muneris, expediam, et calamo properante docebo“; II 1140-1141: „His animadversis, jam quae memoravimus ante // Prona tibi fient, ac debita tempora disces“; II 1201-1202: „Praeterea, has ipsas si mente revolvere causas // Haud pigeat, disces [...]“; III 501: „,...] ratione docebo“; III 556: „[...] paucis adverte, docebo.“ 
Man darf bezweifeln, dass Newtons bahnbrechende Erkenntnisse im Bereich der Mathematik, Astronomie und Optik im Jahre 1760 bereits den kleinen Kindern in der Elementarschule vermittelt werden konnten. Boscovich bemüht dieses Bild wohl nur deshalb, um auf die in der Lehrdichtung etablierte Lehrer-SchülerKonstellation anspielen zu können.

Es entspricht ebenfalls der Gattungstradition, dass der Autor als lehrendes Ich auftritt, um die Authentizität des Gesagten $\mathrm{zu}$ erhöhen. ${ }^{28}$ So verweist er etwa auf seine eigenen Beobachtungen, die er mit seinem Teleskop angestellt habe (III 581-585):

Sic ego cum Phoebi radios excluderet omnes

Arte tubus multa insignis, noctemque foveret In gremio, licet in medio sublimis Olympo

Arderet Titan, sudo nitidissima Coelo

Saepe olim aspexi, propioraque sydera terris.

Auch mit seiner Beteuerung Haec ipsa et domina spectabam nuper in urbe (III 603) folgt Boscovich dem beglaubigenden Prinzip des experto credite, das in der lateinischen Lehrdichtung durch Ovid geprägt worden ist (Ars, III 511).

Den Regeln des Genres folgt Boscovich auch bei der Invokation. Immer wieder, d. h. vor allem zu Beginn eines Buches oder neuen Textabschnittes, spricht der Autor die beiden Planetengötter Phoebus und Urania an und bittet sie um weitere Sachinformationen. ${ }^{29}$ Die Anrufung hat somit wie üblich eine (makrooder mikro-)strukturierende Funktion. Gattungstypisch sind auch solche Formeln, in denen der Verfasser die Unmöglichkeit einer vollständigen Aufzählung

28 Vgl. z. B. II 168: „Vidi [...]“; II 269: „Credo equidem [...]“; III 648: „Nam memini [...].“

29 Vgl. z. B. I 922-925: „Quis prior, Uranie, rem tantam, hominumve, Deûmve // Protulerit, memorare libet; tu numine Vatem, // Diva, tuo dubium firma; remque ordine pande: // Nôsti etenim [...]“; III 21-22: „Huc ades, et trepidum praesenti numine vatem // Usque rege, atque novas in carmina suffice vires“; III 383-390: „Queis tamen auxiliis, quave hanc satis ubere vena // Naturae aggrediar partem, atque arcana docebo? // Nunc ades, O! nunc, Phoebe pater, diffunde micantem // Fronte comam, et flavos per eburnea colla capillos: // His opus est: nunc ardentes age concute habenas: // Flammivomis nunc urge acres calcaribus armos // Quadrijugum: undantem e patulis fac naribus ignem // Commixtum nebula, et fumis nigrantibus efflent“; IV 1-6 u. 10-12: „Post serta obductam Phoebi cingentia frontem // Candida, fulgentesque comas, radiumque bicornem // Signiferi oppositis protentum finibus orbis, // Et vim divini jubaris, plenumque, latente // Dimidio quanquam ore, diem, te, Diva Triformis, // Te canimus [...] // [...] // Tu digitos mihi, tu plectri resonantia eburni // Fila move, cantumque rege, et non impare versu // Ipsa tuos praesens da pandere, Diva, labores.“ 
artikuliert. $^{30}$ Im Übrigen erfolgt die Gliederung des Gedichts mithilfe der janusköpfigen, im Genre etablierten Hactenus-Nunc-Formel, so etwa in I 282-285: ${ }^{31}$

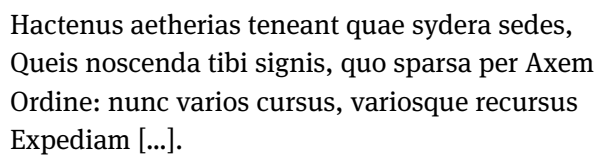

Jeweils am Anfang und Ende eines Buches findet man eine Retrospektive auf das bisher Gesagte sowie eine Vorschau auf das Kommende. ${ }^{32}$ Man muss sich dabei ins Gedächtnis rufen, dass diese Strukturformeln aus der Oralität des homerischen Zeitalters stammen und in einer Epoche, in welcher längere lateinische Lehrgedichte ausschließlich im Medium der Schriftlichkeit rezipiert werden, eigentlich redundant sind. Im typographischen Zeitalter wirken sie vielleicht sogar ein wenig lächerlich, da gedruckte Überschriften der einzelnen Bücher und Kapitel die Funktion solcher Formeln wesentlich besser ausgefüllt hätten.

Wie Boscovich schon in der Vorrede erklärt hat, wird die Wissensvermittlung durch zahlreiche poetische Ornamente, ${ }^{33}$ extrathematische Zusätze und umfangreiche Digressionen aufgelockert. So führt er im Rahmen seiner personifizierenden Darstellung zahlreiche epische Vergleiche $\mathrm{an}^{34}$ und flicht mehrfach Passagen über Ereignisse der antiken Geschichte ein. ${ }^{35}$ Ferner mündet das vierte Buch in einen umfangreichen, persönlich gehaltenen Epilog (IV 832-960), in dem Bosco-

30 Vgl. z. B. I 89-90: „Quis tamen et formas omnes, et nomina pandat // Singula connumerans, certasque ex ordine sedes?“

31 Vgl. auch I 857-860: „Hactenus immensi lustravimus aetheris oras, // Astrorum et positus, variosque ex ordine motus // Diximus: unde jubar nunc accipe, quaeque remittant // Affusos radiorum imbres, ignemque receptum.“

32 Vgl. z. B. II 12-17: „Sic ego velati nigrantem lampada Solis, // Atque laborantem dicturus carmine Lunam, // Materiem satis aptam operi, tantoque labori // Congessi primum, teque astra per ardua vexi, // Nominaque, et positus, variosque per aethera motus // Evolvi enumerans [...]“; V 1-6: „Nunc age cur multo turpatos sanguine vultus // Inficiat, tristem dum Cynthia permeat umbram; // Quid radios fuco saturet, lucemque coloret // Aëriis deflexam auris, et fila retexat, // Jam canere, atque operi supremam impendere curam // Aggredior [...].“

33 So gestaltet er die Buchschlüsse topisch-rhetorisch aus; vgl. z. B. II 1423-1426: „Quo tamen abripior? non hoc mihi Divus Apollo, // Uranie nec movit opus; pars multa laboris // Restat adhuc: fas interea consistere fessum, // Et vocem reparare, novasque resumere vires.“

34 Vgl. z. B. II 120-122: „Non secus ac multa procerum comitante caterva // Per medias si forte vias insignis in ostro // Ingreditur Princeps, dum fervet in aethere Phoebus“; II 272-273: „Nec tantas imo nubes alit Aeolus antro, // Ventorumque ciet gens improba [...].“

35 Vgl. z. B. III 611-625 (Caesar vor der Überquerung des Rubikons); IV 234-256 (Tod des Archimedes bei der Eroberung von Syrakus). 
vich Papst Benedikt XIV. (Pontifikat 1740-1758) sowie den aus dem ,vergilischen“ Mantua $^{36}$ stammenden Kardinal Silvio Valenti Gonzaga (1690-1756, Kardinal seit 1738) verherrlicht, dessen Familie angeblich - wie seine eigene - ursprünglich in Ragusa beheimatet gewesen sei (IV 858). Boscovich berichtet hier zudem über eine geplante Forschungsreise, welche durch eine Überschwemmung des Tibers verhindert worden sei (IV 880-960). Auch das fünfte Buch stellt nahezu in toto eine Digression dar. Da es dem berühmten Kollegen Isaac Newton gewidmet ist und dessen Lehren vorstellt, verweigert der Dichter im Prolog eine Anrufung Apolls und der Musen (V 6-13):

[...] Non hic Pindi pater, atque sororum Docta cohors, non Pegasus aliger, et mendacis Numina Parnassi veniant in carmina. Grande Tu decus Angligenum, atque humanae gloria gentis, Tu majus mihi numen eris, Newtone, repostos Cui primo penetrare aditus, penitusque latentes Sponte dedit vires Natura, arcanaque jura Discere, et attonitum late vulgare per Orbem.

Während die Formel mendacis // Numina Parnassi als ein ferner Reflex der spätantiken Bibeldichtung verstanden werden kann, die sich den paganen Regeln der Invokation verweigert, ${ }^{37}$ verweist das hier verwendete Schlüsselwort Numen, mit dem ein Zeitgenosse als Gottheit und Inspirationsquelle angesprochen wird, ${ }^{38}$ auf Lucans Pharsalia (I 63-66). Dass die gesamte folgende Partie als Hymnus gestaltet ist, bringt Boscovich in einer dazugehörigen Fußnote explizit zum Ausdruck. ${ }^{39}$

Den digressiven Höhepunkt bildet im fünften Buch eine mehr als hundert Verse umfassende Partie (V 914-1024), in der die zuvor beschriebenen astronomisch-physikalischen Vorgänge in eine mythologische Allegorie gekleidet sind. Es handelt sich um ein eigenständiges Epyllion, wie es seit Vergil innerhalb der

36 In IV 854 findet sich ein knapper Verweis auf die Aeneis.

37 Vgl. Paul Klopsch: Einführung in die Dichtungslehren des lateinischen Mittelalters. Darmstadt 1980, S. 7 f., 15, 20-23.

38 Vgl. auch V 89-98: „Ergo novum Coelo delapsum ut Numen ab alto // Te supplex unum veneratur, Apollonis ara // Posthabita, tua scripta terit noctuque, diuque // Evolvens, te in vota vocat, te oracula poscit, // Dona tibi, tibi thura parat studiosa juventus, // Altaque marmoreis meditatur templa columnis. // Huc ades o igitur, vatemque inventa canentem, // Magne, tua, atque almae tua tenvia stamina lucis // Tu praesens moderare, incertos tu rege gressus, // Da facilem venam, et cantum, versusque sonantes.“

39 Vgl. S. 199, Anm. 1: „In sequenti velut hymno [...].“ 
Lehrgedichtstradition häufig begegnet. ${ }^{40}$ In einer Anmerkung erläutert Boscovich hierzu (S. 245f., Anm. 78):

Postremo hujusce episodii fundamentum omne consistit in Veterum mythologia. Phoebus, Diana, Vesta erant apud ipsos Sol, Luna, et Terra [...]; quo casu pronum est fingere, Dianam sua forma superbientem celebrare festa cum ingenti comitatu [...].

Der Leser erfährt, dass es sich bei der mit paganen Gottheiten operierenden Darstellung lediglich um eine Allegorie, d. h. um eine literarische Fiktion handele, die sich an die mythologischen Erzählungen der antiken Dichter (fabulae) anlehne. ${ }^{41}$ Die Grenze zwischen dem facta vermittelnden Sachgedicht und dem mit ficta arbeitenden Epyllion ist somit klar markiert. Letzteres fungiert lediglich als eine poetische Illustration des Ersteren. Boscovich greift hier die rötliche Färbung des Mondes, ein Thema des ersten Buches, auf und erzählt in seiner Geschichte, wie Diana sich aus Wut selbst das Gesicht und die Arme zerkratze, so dass diese von rotem Blut entstellt würden. Mit dem Ende des Epyllions endet auch das gesamte Werk. Einen parathematischen Epilog gibt es nicht.

\section{Antike und nachantike Traditionen und Vorbilder}

Dass Roger Boscovich als lateinischer Lehrdichter des achtzehnten Jahrhunderts mit den antiken Gattungsvorbildern vertraut ist, bedarf kaum der Erwähnung. Insbesondere der Einfluss der vergilischen Georgica ist unübersehbar. ${ }^{42}$ Wie bereits Luca Guzzardi betont hat, ${ }^{43}$ dürfte die berühmte Passage im zweiten Buch der Georgica (II 475-478) die Wahl des Themas motiviert und legitimiert haben:

\footnotetext{
Me vero primum dulces ante omnia Musae, quarum sacra fero ingenti percussus amore, accipiant, caelique uias et sidera monstrent, defectus solis uarios lunaeque labores.
}

40 Vgl. hierzu Hofmann (Anm. 6); ders.: Rezension zu: Brills’ Encyclopaedia of the Neo-Latin World. Hg. von Philip Ford, Jan Bloemendal, Charles Fantazzi. Bd. 1-2. Leiden, Boston 2014. In: Gnomon 90 (2018), S. 22-48 u. 117-141, hier S. 43.

41 Vgl. bei Boscovich auch S. 248, Anm. 78: „Fabulati sunt Veteres [...].“

42 Vgl. hierzu Guzzardi (Anm. 5), S. 16-20; zum Einfluss Vergils auf die neulateinische Lehrdichtung vgl. Ludwig (Anm. 7).

43 Guzzardi (Anm. 5), S. 17 f. 
Auch die meisten der bereits genannten, die Gattungstradition maßgeblich prägenden Formeln und Strukturmerkmale, welche man bei Boscovich finden kann, gehen letztlich auf Vergil zurück: so etwa die Hactenus-Nunc-Struktur, ${ }^{44}$ der Einstieg über indirekte Fragen ${ }^{45}$ und die programmatische Paraphrase rerum causas (latentes) ${ }^{46}$. Ferner verarbeitet Boscovich nicht nur einzelne vergilische Junkturen und Klauseln, sondern zitiert sogar ganze Verse. ${ }^{47}$ Dass der allegorische Schluss seines Gedichts (V 914-1024) durch das Aristaeus-Epyllion (Georg., IV 315-558) geprägt ist, liegt ebenfalls auf der Hand. ${ }^{48}$

Unter den antiken Dichtern stellt Vergil jedoch nicht das einzige Vorbild dar. Angesichts des astronomischen Themas muss man hier zunächst an die Phainomena des Aratos von Soloi und deren lateinische Übersetzungen (Germanicus und Avienus) denken. Es dürfte kein Zufall sein, dass Boscovich in seiner Vorrede mehrfach von phaenomena spricht. ${ }^{49}$ Nachweisbar ist ferner eine Beeinflussung durch Lukrez. Wie dieser, ${ }^{50}$ so will auch Boscovich mithilfe seiner wissenschaftlichen Erklärungen die Menschen von falschen Ansichten befreien und ihnen so die Furcht nehmen (II 1360-1361): Excutimusque metum monitis, causasque latentes // Pandimus, atque animi motus cohibemus inanes. Zudem

44 Vgl. Vergil, Georg., II 1-2: „Hactenus aruorum cultus et sidera caeli; // nunc te, Bacche, canam [...]“; vgl. hiermit Boscovich III 1-6: „Hactenus in medio cur Sol, cur aurea quondam // Deficiat cursu Phoebe, et ferrugine multa // Involvat nitidos puro vel in aethere vultus: // Nunc age, velati nigrantem lampada Solis // Quae tenuis circum lux ambiat; unde repente // Exiliant toto radiantia sydera Coelo.“

45 Vgl. Vergil, Georg., I 1-5: „Quid faciat laetas segetes, quo sidere terram // uertere, Maecenas, ulmisque adiungere uitis // conueniat, quae cura boum, qui cultus habendo // sit pecori, apibus quanta experientia parcis, // hinc canere incipiam [...]." Vgl. auch Boscovichs Vorrede (Ad Lectorem), S. i: „Secundo libro ipsam eclipsium naturam aggressus, illud explicavi, unde proveniant primo quidem Solis, tum Lunae defectus, quo ordine, et quibus temporum intervallis [...].“

46 Vgl. Vergil, Georg., II 490: „felix qui potuit rerum cognoscere causas.“ Vgl. Boscovich, II 1362-1363: „Felices anni, felicia saecula, causas // Tantarum queis posse datum cognoscere rerum?“; III 11-12: „[...] causasque latentes // Protinus evolvam, primaque ab origine pandam.“ 47 So zitiert er in II 1401-1403 Vergil, Georg., I 466-468.

48 Zur Epyllientradition in neulateinischen Lehrgedichten vgl. Hofmann (Anm. 6); ders. (Anm. 40), S. 43.

49 Vgl. Vorrede (Ad Lectorem), S. i: „Tertio libro sum persecutus phaenomena [...]“; S. ii: „Quarto libro pertractavi phaenomena [...]“; S. ii: „Quinto demum libro illud exposui, cur Luna in totalibus eclipsibus saepe admodum appareat rubro colore affecta, cujus itidem phaenomeni satis uberem explicationem alibi non reperi [...].“

50 Vgl. z. B. Lukrez, III 37: „et metus ille foras praeceps Acheruntis agendus.“ 
geht der Autor explizit auf Lukrezens Ausführungen zur Sonnen- und Mondfinsternis (V 751-779) ein. Hier schreibt er (II 1362-1374):

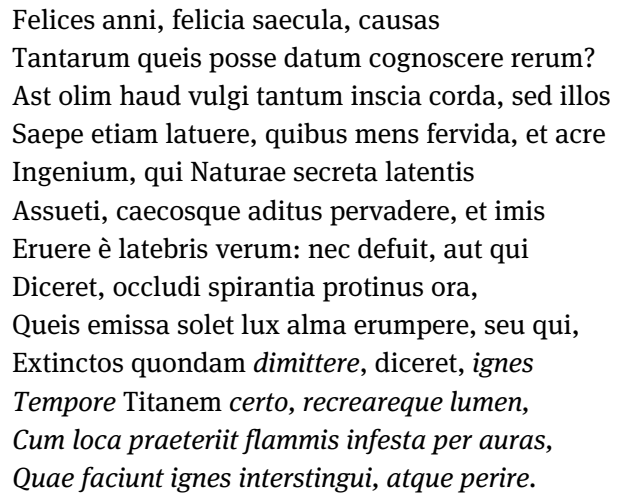

Boscovich spielt hier auf einige griechische Naturphilosophen an (II 1368-1370) und zitiert anschließend (auch bei ihm typographisch abgesetzt) einige Verse aus De rerum natura (V 758-761). Ferner schildert er am Ende des vierten Buches die Überschwemmung des Tibers, welche im Jahre 1751 die Stadt Rom heimgesucht hat (IV 880-960). Die Darstellung dieser Naturkatastrophe erinnert unmittelbar an Lukrezens sechstes Buch, an dessen Ende beschrieben wird, wie Athen von der Pest verheert wird (VI 1138-1286).

Nicht nur das astronomische Thema, sondern auch die Anzahl der Bücher (5 libri) sowie deren ungefähr vergleichbare Umfänge verweisen zudem auf Manilius. Als sich Boscovich 1760 in London aufhielt, waren die Astronomica dort sehr präsent, da Richard Bentley den Text 1739 kritisch ediert hatte. Boscovichs mehrfache Klagen über die Schwierigkeit bzw. Unmöglichkeit, den Stoff in Versen wiederzugeben, ${ }^{51}$ sind nicht denkbar ohne die berühmte Passage im dritten Buch der Astronomica (III 31-42), in der Manilius ähnliche Gedanken formuliert hat.

Man könnte sich mit allen diesen Parallelen begnügen und Boscovich als typischen Vertreter neulateinischer Poesie etikettieren, welcher ganz auf die Antike fixiert sei. Dennoch lohnt es sich, abschließend einen Blick auf die nachantike Tradition des Lehrgedichts zu werfen. Dass Boscovich auch zeitgenössische und insbesondere jesuitische Gattungsvertreter gekannt hat, ist selbstverständlich. In seinem Poem rühmt er etwa Carlo Noceti, dessen Gedichte De Aurora Boreali und De Iride er selbst zusammen mit eigenen Anmerkungen dreizehn Jahre zuvor

51 Vgl. I 487-491 u. IV 257-262 sowie S. 21 (Anm. 29). 
veröffentlicht hat. ${ }^{52}$ Hingegen dürfte ihm die breite Lehrgedichtstradition des Mittelalters und der frühen Renaissance nicht bekannt gewesen sein. ${ }^{53}$ Und doch zeigen sich hier fünf Strukturanalogien:

Als Erstes zu nennen ist die Existenz von Paratexten: Im Gegensatz zu fast allen antiken Lehrgedichten arbeiten die nachantiken Gattungsvertreter zumeist mit Prosa-Einleitungen, in denen über Inhalt, Intention und Publikum des jeweiligen Werkes gesprochen wird. Boscovich folgt dieser nachantiken, erst im Mittelalter aufkommenden Tradition, indem er sein Werk durch eine lange, an den anonymen Leser adressierte Prosa-Praefatio einleitet.

Zweitens fällt die Dominanz des begleitenden Kommentars auf. Boscovichs Text steht auf zwei Beinen, der verdunkelnden Poesie und der sie erhellenden Prosa. Dass Lehrgedichte nicht nur durch einzelne Glossen, sondern sogar durch kohärente Kommentare erklärt werden, lässt sich punktuell bereits in der Antike beobachten (berühmt sind etwa die Scholia zu den Phainomena des Arat). Doch erst im Mittelalter entwickelt sich die Praxis, dass die Lehrdichter selbst ihre eigenen Carmina mit umfangreichen Prosa-Kommentaren versehen. In dieser Tradition steht Boscovich. Es bedeutet lediglich eine technische Neuerung des typographischen Zeitalters, dass sein Kommentar nicht, wie im Mittelalter, am Rand oder im Rahmen eines textus-inclusus-Schemas, sondern in Fußnoten organisiert ist.

Eine dritte Strukturanalogie zeigt sich in der sozialen bzw. beruflichen Situation des Autors. Die antiken Lehrgedichte werden von Personen geschrieben, deren Alltag nicht durch die Vermittlung der im jeweiligen Gedicht behandelten Inhalte geprägt ist. Manche von ihnen - wie etwa Lukrez und Vergil - besitzen immerhin eine fundierte Sachkompetenz, andere wiederum - wie etwa die hellenistischen Dichter Arat und Nikander - haben sich das gewählte Thema nur oberflächlich angelesen. ${ }^{54}$ In beiden Gruppen findet sich allerdings kein professioneller Lehrer. Auch einem Autor wie Vergil, dem man eine große Ernsthaftigkeit bei der

52 Vgl. III 433-442: „Dum celebrat plectro Auroram, fidibusque canoris, // Aethereos nuper Nicetas edidit ortus, // Et causas rutili victuro carmine rhombi: // Musarum decus, et nostri clarissima Pindi // Gloria Nicetas, quo non praestantior alter // Aut fuit Arcadicis, aut olim exsurget in arvis // Scrutari Sophiae sedes, atque abdita quaeque // Eruere, et Latiis proferre exculta Camoenis. // Pauca modo expediam: aeternum, si plura requiris, // Perlege opus vigil; haud equidem vigilasse pigebit." Vgl. hierzu auch seine entsprechende Anmerkung (S. 129, Anm. 22).

53 Vgl. Thomas Haye: Das lateinische Lehrgedicht im Mittelalter. Analyse einer Gattung. Köln, Leiden 1997 (Mittellateinische Studien und Texte 22).

54 Vgl. hierzu Haskell (Anm. 5), S. 209 (mit Verweis auf Cicero, De oratore I 16,69). 
Vermittlung des landwirtschaftlichen Themas sowie eine erhebliche Sachkompetenz unterstellen darf, hat nie an einer agrarwissenschaftlichen Fakultät unterrichtet. Anders hingegen die berühmten Lehrdichter des Mittelalters: Diese sind - wie der Mathematik-Professor Boscovich - auch außerhalb des literarischen Rahmens didaktisch aktiv. Zwar müssen solche Gedichte nicht immer unmittelbar für den Unterricht geschaffen worden oder aus ihm hervorgegangen sein, doch lässt sich wie bei Boscovich - stets ein direkter Zusammenhang zwischen der poetischen Rolle des Lehrdichters und der extrapoetischen Existenz des Autors beobachten. Die Frage der fachlichen Kompetenz sowie der Ernsthaftigkeit sachlicher Belehrung beantwortet sich in solchen Fällen von selbst.

Eine vierte Strukturanalogie zur mittelalterlichen Tradition greift man in der Textgeschichte. Die antiken Lehrgedichte sind literarische Produkte, deren Verfasser eine finale und $\mathrm{zu}$,publizierende‘ Fassung anstreben. Hingegen arbeiten viele mittelalterliche Lehrdichter über Jahrzehnte an ihren Carmina. Gerade aufgrund der Verankerung im Unterricht führen Autor und Text nicht selten ein symbiotisches Dasein. In der Folge kursieren unterschiedliche Fassungen und Redaktionsstufen nebeneinander. Dass ein Lehrgedicht nicht etwa ein finales Produkt, sondern ein ewiger Prozess sein kann, der zudem zwischen den Medien der Oralität und der Skripturalität pendelt, beobachtet man auch im Falle Boscovichs.

Eine fünfte und letzte Analogie zeigt sich in der religiösen Fundierung: Insbesondere im Bereich der Astronomie und Astrologie müssen sich die nachantiken, d. h. per definitionem christlichen Autoren gegenüber dem paganen Mythos positionieren. Die Lehrdichter des Mittelalters und der Renaissance distanzieren sich in der Regel explizit von der heidnischen Götterwelt, halten jedoch unter Hinweis auf die poetische Tradition an den Planetenbezeichnungen und den mit diesen verknüpften Personifikationen und mythischen Erzählungen fest. Beliebt ist zudem die Betonung des allegorischen Charakters: Durch die Allegorie kann der Lehrdichter weiterhin das illustrative Potential der paganen Sagen nutzen und sich gleichzeitig von ihnen ideologisch abgrenzen. Wenn Boscovich am Ende seines Werkes zur Illustration astronomischer Vorgänge ein mythologisches Epyllion präsentiert und in penetranter Deutlichkeit betont, dass es sich dabei lediglich um erfundene Geschichten der heidnischen Antike handele, unterscheidet er sich kaum von dem spätmittelalterlichen Dichter Simon von Couvin, der in seinem um 1350 verfassten Werk De iudicio Solis in conviviis Saturni ganz ähnlich agiert. $^{55}$

55 Vgl. Thomas Haye: Simon von Couvin: De iudicio Solis in conviviis Saturni. Einleitung und kritische Edition. In: Wiener Studien 127 (2014), S. 217-313. 
Im Ergebnis darf man festhalten, dass Boscovichs Opus De Solis ac Lunae defectibus zwar eng (insbesondere auf sprachlicher Ebene) an den antiken Modellen orientiert ist, jedoch zusätzlich einige Merkmale aufweist, die erst die nachantike Gattungsentwicklung hervorgebracht hat. Dass der Autor für seine Beiträge zum aktuellen wissenschaftlichen Diskurs auch die altehrwürdige, doch zu seiner Zeit bereits etwas angestaubte Form des Lehrgedichts gewählt hat, ist wohl primär mit dem jesuitischen Milieu sowie dem eigenen literarischen Ehrgeiz zu erklären. Als Wissenschaftler spielt Boscovich in derselben fachlichen Liga wie Leibniz und Newton, doch haben sich diese beiden eben nicht der Form des Lehrgedichts bedient. Belächelt worden ist Boscovich deshalb nicht, da seine akademische Kompetenz unbestritten war. Im Bereich der Dichtung wollte er sich als poeta ambidexter profilieren, ${ }^{56}$ und gerade im römischen Milieu hat man diese Doppelleistung auch zu würdigen gewusst. Dennoch dürfte in der Mitte des achtzehnten Jahrhunderts allen Beteiligten, d. h. auch dem Lehrdichter selbst, bewusst gewesen sein, dass die Welt der Wissenschaft längst prosaisch geworden war.

56 Vgl. Haskell (Anm. 5), S. 209. 